The authors concluded that, in view of the high morbidity and mortality in patients with MRSA-positive isolates, specific antibiotic prophylaxis against MRSA should be considered in patients undergoing major amputation.

FROM: Grimble SA, Magee TR, Galland RB. Methicillin-resistant Staphylococcus aureus in patients undergoing major amputation. Eur J Vasc Endovasc Surg 2001;22:215-218.

\section{Staphylococcus aureus Bacteremia Associated With Pacemakers and Implantable Defibrillators}

Although cardiac device infections (CDIs) are a devastating complication of permanent pacemakers or implantable cardioverter-defibrillators, the incidence of CDI in patients with bacteremia is not well defined. Chamis and coinvestigators, from the Duke University Medical Center, Durham, North Carolina, conducted a study to determine the incidence of CDI among patients with permanent pacemakers or implantable cardioverter-defibrillators who develop Staphylococcus aureus bacteremia (SAB).

A cohort of all adult patients with $\mathrm{SAB}$ and permanent pacemakers or implantable cardioverter-defibrillators over a 6-year period was evaluated prospectively. The overall incidence of confirmed CDI was 15 of $33(45.4 \%)$. Confirmed CDI occurred in 9 of the 12 patients (75\%) with early SAB ( $<1$ year after device placement). Fifteen of 21 patients $(71.5 \%)$ with late SAB ( $\geqslant 1$ year after device placement) had either confirmed $(6 / 21,28.5 \%)$ or possible $(9 / 21,43 \%)$ CDI. In $60 \%$ of the patients $(9 / 15)$ with confirmed CDI, no local signs or symptoms suggesting generator pocket infection were noted.

The authors concluded that the incidence of CDI among patients with $\mathrm{SAB}$ and cardiac devices is high. Neither physical examination nor echocardiography can exclude the possibility of CDI. In patients with early SAB, the device is usually involved, and approximately $40 \%$ of these patients have obvious clinical signs of cardiac-device involvement. Conversely, in patients with late $\mathrm{SAB}$, the cardiac device rarely is the initial source of bacteremia, and there is a paucity of local signs of device involvement. The cardiac device is involved, however, in $28 \%$ or more of these patients.

FROM: Chamis AL, Peterson GE, Cabell CH, Corey GR, Sorrentino RA, Greenfield RA, et al. Staphylococcus aureus bacteremia in patients with permanent pacemakers or implantable cardioverter-defibrillators. Circulation 2001;104:1029-1033.

\section{Influenza Outbreak in a Transplant Unit}

Although there is a strong body of evidence in favor of influenza virus immunization in solid-organ recipients, little attention has been devoted to other reservoirs, such as the patients' relatives and, at the time of hospital admission, healthcare workers. Malavaud and coinvestigators from
France report on an analysis of the epidemiology of an outbreak of nosocomial influenza $A$ in a solid-organ transplant unit. Four cases of influenza A virus infection were reported during a short 4-day outbreak in a 12 single-room transplant unit. None of the patients had been immunized against influenza. Three patients had not been visited by their relatives between admission and influenza infection. Three nurses, among the 27 healthcare workers, presented with clinical flu symptoms at times consistent with nosocomial transmission.

Because the prevention of influenza infection by vaccination warrants a global strategy to target the different reservoirs, the authors suggest that the modern policy of vaccinating solid-organ patients should be extended both to their relatives and to the healthcare workers of transplant units.

FROM: Malavaud S, Malavaud B, Sandres K, Durand D, Marty N, Icart J, et al. Nosocomial outbreak of influenza virus A (H3N2) infection in a solid organ transplant department. Transplantation 2001;72:535-537.

\section{Risk Factors for Mortality and Nosocomial Infections in Elderly Cardiac Surgery Patients}

Rady and coinvestigators, from the Cleveland Clinic Foundation, conducted an inception cohort study to determine perioperative predictors of morbidity and mortality in patients $\geqslant 75$ years of age after cardiac surgery. The setting was a 54-bed, tertiary-care, cardiothoracic intensive care unit (ICU); the study included all patients aged $\geqslant 75$ years admitted over a 30 -month period for cardiac surgery. Data were collected on preoperative factors, operative factors, postoperative hemodynamics, and laboratory data obtained on admission and during the ICU stay.

Postoperative death, frequency rate of organ dysfunction, nosocomial infections, length of mechanical ventilation, and ICU stay were recorded. During the study period, 1,157 (14\%) of 8,501 patients $\geqslant 75$ years of age had a morbidity rate of $54 \%$ ( $625 / 1,157$ patients) and a mortality rate of $8 \%$ (90/1,157 patients) after cardiac surgery. Predictors of postoperative morbidity included preoperative intraaortic balloon counterpulsation, preoperative serum bilirubin of $>1.0 \mathrm{mg} / \mathrm{dL}$, blood transfusion requirement of $>10$ units of red blood cells, cardiopulmonary bypass time of $>120$ minutes (aor tic cross-clamp time of $>80$ minutes), return to operating room for surgical exploration, heart rate of $>120$ beats/min, requirement for inotropes and vasopressors after surgery and on admission to the ICU, and anemia beyond the second postoperative day.

Predictors of postoperative mortality included preoperative cardiac shock, serum albumin of $<4.0 \mathrm{~g} / \mathrm{dL}$, systemic oxygen delivery of $<320 \mathrm{~mL} / \mathrm{min} / \mathrm{m}^{2}$ before surgery, blood transfusion requirement of $>10$ units of red blood cells, cardiopulmonary bypass time of $>140$ minutes (aortic cross-clamp time of $>120$ minutes), subsequent return to the operating room for surgical exploration, mean arterial pressure of $<60 \mathrm{~mm} \mathrm{Hg}$, heart rate of $>120$ beats $/ \mathrm{min}$, cen- 
tral venous pressure of $>15 \mathrm{~mm} \mathrm{Hg}$, stroke volume index of $<30 \mathrm{~mL} / \mathrm{min} / \mathrm{m}^{2}$, requirement for inotropes, arterial bicarbonate of $<20 \mathrm{mmol} / \mathrm{L}$, plasma glucose of $>300 \mathrm{mg} / \mathrm{dL}$ after surgery, and anemia beyond the second postoperative day. During the study period, the study cohort used 6,859 (21.5\%) ICU patient-days out of a total 31,867 ICU patientdays. Nonsurvivors used 2,023 (30\%) ICU patient-days, and patients with morbidity used 5,903 (86\%) ICU patient-days.

The authors concluded that severe underlying cardiac disease (including shock, requirement for mechanical circulatory support, hypoalbuminemia, and hepatic dysfunction), intraoperative blood loss, surgical reexploration, long ischemic times, immediate postoperative cardiovascular dysfunction, global ischemia and metabolic dysfunction, and anemia beyond the second postoperative day predicted poor outcome in the elderly after cardiac surgery. Postoperative morbidity and mortality disproportionately increased the utilization of intensive care resources in elderly patients. Future efforts should focus on preoperative selection criteria, improvement in surgical techniques, perioperative therapy to ameliorate splanchnic and global ischemia, and avoidance of anemia to improve the outcome in the elderly after cardiac surgery.

FROM: Rady MY, Ryan T, Starr NJ. Perioperative determinants of morbidity and mortality in elderly patients undergoing cardiac surgery. Crit Care Med 2001;29(suppl 9):\$163-S172.

\section{Clinical Diagnosis of Influenza}

Successful treatment of influenza depends on an accurate diagnosis of the illness and prompt intervention. Unfortunately, there is a lack of data comparing clinical diagnosis versus laboratory diagnostic techniques.
Zambon and colleagues from the Central Public Health Laboratory in London conducted a study to compare the clinical diagnosis of community cases of influenza with various laboratory diagnostic techniques, including multiplex, reverse transcription polymerase chain reaction. Clinical diagnosis, viral isolation, hemagglutinin inhibition serology, and multiplex reverse transcription polymerase chain reaction were used to diagnose influenza in patients enrolled in international phase 3 studies designed to investigate the efficacy and safety of an antiinfluenza drug (inhaled zanamivir). Patients clinically diagnosed with influenza were enrolled at centers across North America and Europe.

A total of $791(77 \%)$ of 1,033 patients with laboratory results from all three methods were confirmed positive for influenza by one or more test results. For 692 patients (67\%), the results of all three tests agreed. Total symptom scores at baseline showed a significant association toward greater severity of symptoms with an increasing number of positive test results $(P<.001)$. An increasing number of positive test results also showed a significant correlation with a longer time to alleviation of symptoms of influenza in the placebo group $(P=.001)$.

The authors conclude that, during a time when influenza was known to be circulating and clinical diagnostic criteria were applied, diagnosis of influenza in these trials was accurate in approximately $77 \%$ of adults on clinical grounds alone. This highlights the need for primarycare physicians to be alerted to circulating influenza and to be aware cough and fever are the most predictive symptoms.

FROM: Zambon M, Hays J, Webster A, Newman R, Keene O. Diagnosis of influenza in the community: relationship of clinical diagnosis to confirmed virological, serologic, or molecular detection of influenza. Arch Intern Med 2001;161:2116-2122. 